\title{
O impacto do Ensino Remoto Emergencial, no contexto da pandemia da COVID-19, na saúde mental dos docentes universitários
}

\section{The impact of Emergency Remote Teaching, in the context of the COVID-19 pandemic, on the mental health of university teachers}

\author{
Luana dos Passos Bispo ${ }^{1 *}$, Paulo César Marques de Andrade Santos ${ }^{1}$, Tarcísio Fulgêncio Alves \\ da Silva ${ }^{1}$
}

\begin{abstract}
RESUMO
Devido a diversidade e a complexidade das atividades realizadas pelos docentes universitários, o ambiente acadêmico tem sido considerado um local causador de estresse em virtude de fatores organizacionais e psicossociais do trabalho. Considerando a existência destes aspectos aliados ao atual cenário de crise devido a pandemia da COVID-19, percebe-se um processo árduo de reinvenção docente. Surge assim o Ensino Remoto Emergencial (ERE) junto com a exigência de uma adequação da força de trabalho dos professores para o teletrabalho. Diante de tal cenário, os educadores se expõem a diversas situações, que repercute no adoecimento, principalmente relacionado à saúde mental. Sendo assim, esse estudo tem como objetivo compreender as dificuldades encontradas pelos docentes universitários para execução do ERE. A presente pesquisa trata-se de uma revisão biblbiográfica, de caráter descritivo e exploratório. Pôde-se perceber a importância da necessidade do acompanhamento das condições estruturais, tecnológicas e pedagógicas que os docentes possuem para realização do ensino remoto, bem como do cuidado com a saúde mental visando o despertar para a necessidade de políticas públicas de preservação e promoção da saúde dos educadores.
\end{abstract}

Palavras-chave: Coronavírus; Professor; Educação; Saúde Mental; Teletrabalho.

\begin{abstract}
Due to the diversity and complexity of activities carried out by university professors, the academic environment has been considered a place that causes stress due to organizational and psychosocial factors at work. Considering the existence of these aspects allied to the current crisis scenario due to the COVID19 pandemic, there is an arduous process of teaching reinvention. Thus, Emergency Remote Education (ERE) emerges along with the demand for an adequacy of the teachers' workforce for teleworking. Faced with such a scenario, educators are exposed to different situations, which have repercussions on illness, mainly related to mental health. Therefore, this study aims to understand the difficulties encountered by university professors to perform the ERE. The present research is a bibliographic review, of a descriptive and exploratory nature. It was possible to perceive the importance of the need to monitor the structural, technological and pedagogical conditions that teachers have to carry out remote teaching, as well as the care with mental health, aiming at awakening to the need for public policies for the preservation and promotion of health of educators.
\end{abstract}

Keywords: Coronavirus; Teacher; Education; Mental Health; Telecommuting.

\footnotetext{
${ }^{1}$ Universidade de Pernambuco - UPE.

*E-mail: luana.passos@upe.br
} 


\section{INTRODUÇÃO}

Devido a fatores psicossociais e organizacionais do trabalho, o ambiente acadêmico tem sido considerado um local gerador de estresse para os docentes universitários. A precarização nas condições de trabalho, a frequente exposição a agentes de risco, a desvalorização da imagem do professor, os baixos salários, a falta de recursos humanos e materiais para desenvolverem suas atividades e o esgotamento físico, causado pelo aumento do ritmo do trabalho, está afetando diretamente a saúde dos educadores. (SOARES; MAFRA; FARIA, 2019).

Considerando estes aspectos e em virtude da diversidade e complexidade das tarefas realizadas por esses profissionais, estudos demonstraram que a probabilidade de professores desenvolverem estresse, depressão e ansiedade é duas vezes maior quando comparados às demais profissões (SANTOS; SILVA; BELMONTE, 2021). Sendo assim, a docência no Brasil está envolvida, em inúmeros fatores que contribuem para a insatisfação deste público, pois além das atividades de ensino, pesquisa e extensão os professores agregam outras funções de caráter administrativo. (SOARES; MAFRA; FARIA, 2019).

Estes fatores aliados ao atual cenário de crise gerado pela pandemia da COVID19, exigiu que mudanças fossem realizadas no sistema educacional, além de demandar um processo árduo de reinvenção docente, que deveria ser adaptado para uma educação em formato remoto, que fosse presente e acessível as tecnologias de rede, possibilitando a comunicação on-line, mas que não considerou os problemas existentes nas condições estruturais, trabalhistas e na formação destes profissionais. (PEREIRA; SANTOS; MANENTI, 2020).

Segundo o Ministério da Saúde (2020), a COVID-19 é uma doença provocada pelo novo coronavírus, cientificamente identificado como SARS-COV-2, que causa infecções respiratórias. O mesmo foi descoberto em 31 de dezembro de 2019 após casos registrado na cidade de Wuhan, na China. Em 30 de janeiro de 2020 a Organização Mundial da Saúde - OMS (2020) declarou que a pandemia da COVID - 19 se constitui em uma Emergência de Saúde Pública de Importância Internacional (ESPII), o mais alto nível de alerta emergencial da Organização, conforme previsto no Regulamento Sanitário Internacional. (BARROS DOS SANTOS JÚNIOR; MONTEIRO, 2020). 
Com o intuito de atenuar a disseminação da doença e suas consequências, o Brasil, atendendo às recomendações da Organização Mundial da Saúde (OMS), adotou diversas medidas individuais e coletivas, a exemplo do afastamento social como restrição de contato (GOMES et al., 2021). A partir desse processo, iniciam-se as mudanças nas Instituições de Ensino com a manifestação do Ministério da Educação (MEC), através da Portaria n ${ }^{\circ} 343$, do dia 17 de março de 2020, onde o mesmo informa sobre a substituição das aulas presenciais por aulas em meios digitais, enquanto durar a situação de pandemia da COVID-19, para Instituição de Educação Superior (IES) integrante do Sistema Federal de Ensino. Posteriormente, tal Portaria recebeu ajustes e acréscimos por meio das Portarias 344 e 345/2020. (BRASIL, 2020). Sendo assim, visando cumprir as recomendações do MEC, as Instituições de Ensino fecharam temporariamente e passaram a avaliar as possibilidades de utilizar estratégias das Tecnologias de Informação e Comunicação (TIC). (BARROS DOS SANTOS JUNIOR; MONTEIRO, 2020).

Levando em conta os aspectos citados e visando ampliar as pesquisas relacionadas ao trabalho docente e saúde mental, este estudo tem como objetivo avaliar o impacto da implementação do Ensino Remoto Emergencial (ERE), considerando que o mesmo, da forma como foi instituido, pode comprometer a saúde mental dos docentes universitários. Dessa forma, considerando que existe um número reduzido de estudos que enfatizam o trabalho dos professores do ensino superior, bem como a repercussão deste na saúde mental dos profissionais, torna-se necessário desenvolver mais pesquisas direcionados a essa categoria tendo em vista a responsabilidade que os mesmos possuem na formação de futuros profissionais. (COSTA et al., 2021).

Considerando o exposto acima, a presente pesquisa trata de um artigo de revisão bibliográfica. Para isso, foi realizado um levantamento no banco de dados google acadêmico, Scielo e portal Capes, com os descritores saúde mental de docentes universitários, adoecimento de professores universitários durante a pandemia. $\mathrm{O}$ artigo foi estruturado em dois tópicos: o primeiro aborda o trabalho docente no ensino remoto emergencial e o segundo aborda a saúde mental dos docentes universitários.

\section{REFERENCIAL TEÓRICO}

\section{TRABALHO DOCENTE NO ENSINO REMOTO EMERGENCIAL}


A pandemia da COVID-19, tem se propagado de forma avassaladora, causando diversos impactos na sociedade, em especial na área da educação. Os esforços estão direcionados para a adoção de medidas de isolamento de toda população na busca de conter a transmissibilidade do vírus e reduzir os índices de mortalidade. Sendo assim, após as recomendações da OMS e publicação das portarias do Ministério da Educação, foi necessário que as Instituições de Nível Superior e seus docentes criassem estratégias para organização das práticas educativas, com o objetivo de proporcionar um ensino inovador. Diante de tal cenário, os educadores ficaram expostos a diversas situações, além de sofrerem pressão das instituições. (SILVA; ESTRELA; LIMA; ABREU, 2020).

No Brasil, o contexto da pandemia resultou na suspensão de aulas presenciais no setor público e privado. No início de maio de 2020, cerca de $89,4 \%$ das universidades federais estavam com as atividades de ensino suspensas (BRASIL, 2020a). 91\% dos estudantes no mundo tiveram as atividades presenciais interrompidas (GUSSO et al., 2020). Essa interrupção das aulas modificou a rotina dos profissionais da área de educação, em especial, dos docentes que precisaram reorganizar estratégias para ministrar suas aulas de forma on-line. (SILVA; BATISTA; TROTTA, 2020).

Sendo assim, o ensino presencial precisou ser transposto para os meios digitais. Inicia-se assim o Ensino Remoto Emergencial (ERE) que compreende um conjunto de atividades emergenciais que as escolas e Universidades estão planejando e executando por meio de ferramentas virtuais ou outros meios disponíveis, visando minimizar os impactos da suspensão das aulas presenciais, em virtude dos planos de contingência do Ministério da Saúde, Secretarias Estaduais e Municipais de Saúde para evitar a disseminação do novo coronavírus. Essa nova forma de ensino difere, portanto, de uma Educação a Distância (EAD) que dispõe de uma organização, planejamento e lógica de funcionamento própria. (CARVALHO; ARAÚJO, 2020).

Podemos, portanto, dizer que o ERE é uma forma de ensino que pressupõe o distanciamento do ambiente escolar de professores e alunos e foi adotado de forma temporária nos diferentes níveis de ensino por instituições educacionais do mundo inteiro para que as atividades escolares não fossem interrompidas. No Brasil, essa forma de ensino foi admitida pelo Conselho Nacional de Educação (CNE) e ratificada pelo MEC em substituição a modalidade de ensino presencial, enquanto perdurar a Pandemia. Assim, é preciso diferenciar, neste momento, que a maior parte das instituições de ensino não está fazendo Educação a Distância, e sim ERE. (BEHAR, 2020). 
Muito embora o ERE utilize os meios de transminssão e recepção de dados semelhantemente a Educação a Distância $(\mathrm{EaD})$, elas são distintas. $\mathrm{A} \mathrm{EaD}$ é uma modalidade de ensino que aplica os princípios pedagógicos mediados pelas tecnologias de informação e comunicação, sem, contudo, excluir as atividades presenciais. Está organizada segundo metodologia, sistema de avaliação e gestão que atendem aos princípios previstos na Lei de Diretrizes e Bases da Educação Nacional (LDB 9394/96), e as várias estratégias do Plano Nacional de Educação. Sendo assim, a EAD dispõe de uma organização, planejamento e lógica de funcionamento própria. O ERE é um arranjo criado para atender a uma excepcionalidade. Como não foi criada com bases e projeções pedagógicas, os seus resultados são desconhecidos. (CARVALHO; ARAÚJO, 2020).

Nesse novo formato, a aula ocorre de forma síncrona, que de forma geral, segue as mesmas orientações do ensino presencial, por meio de aulas expositivas realizadas a partir de sistema de webconferência, bem como disponibilização de videoaula. Além desses momentos, as atividades seguem durante a semana, de forma assíncronas no espaço de um ambiente virtual de aprendizagem (AVA). Dessa forma, a presença física do professor e do aluno no espaço da sala de aula presencial foi substituída por encontros on-line na sala de aula virtual. (BEHAR, 2020).

Considerando estes aspectos, foi necessário que os profissionais da educação repensassem na possibilidade de utilizar métodos de ensino pouco tradicionais, considerando os modelos utilizados no ensino presencial (AVELINO; MENDES, 2020). Sendo assim, acostumados a metodologia empregada anteriormente, os docentes tiveram que deixar de lado a realidade que estavam familiarizados. Mas, para esse novo processo, muitos educadores não estavam preparados. (BEHAR, 2020).

Essa rápida adesão ao ERE para atender à demanda urgente do momento se tornou mais um grande desafio para os professores. Em meio às adversidades impostas pelo contexto completamente atípico, marcado por cobranças, medo, incertezas, dúvidas e expectativas, destinou-se aos docentes a necessidade de aprimorar estratégias pedagógicas, resguardando, ao mesmo tempo, a qualidade do ensino (SANTOS; SILVA; BELMONTE; 2021). Nesse sentido, a pandemia tem exigido que os trabalhadores se adaptem a novas situações de trabalho. Muitos estão desempenhando suas atividades laborais de forma remota, onde muitos não possuem um ambiente com condições ideais para realizarem esse trabalho em suas casas (FANTINI et al., 2020). Esses aspectos estão 
repercutindo no adoecimento, principalmente relacionado à saúde mental. (SILVA; ESTRELA; LIMA; ABREU, 2020).

Muitos são os fatores que dificultam a realização do trabalho docente nessa nova realidade. Entre eles, pode-se destacar a ausência de metodologia que produza maior participação dos estudantes nas aulas sícronas. A falta de uma metodologia adequada é fruto de uma didática que não produz os efeitos esperados nas relações de ensino $\mathrm{x}$ aprendizagem, ocasionado pela (I) falta de formação para utilização das TICs; (II) escassez e precariedade da internet; e, (III) falta de infraestrutura e de equipamentos. Esses fatores afetam tanto o corpo docente quanto o corpo discente prejudicando a compreensão e aprendizado de conteúdos importantes presentes tanto no currículo básico quanto no profissionalizante. (COSTA et al., 2021).

Estes fatores reunidos produzem efeitos já observados no curto prazo nas aulas remotas como a quantidade excessiva de aulas expositivas, sem nenhuma ou pouca participação dos alunos. Essa característica jé é um indicativo de que boa parte dos discentes estão desmotivados com o ERE. A forma como vem sendo conduzida as atividades está causando um distanciamento entre os atores envolvidos, pois devido a falta ou limitação dos recursos, as câmeras ficam desligadas durante as aulas on-line gerando sentimento nos professores de que estão sozinhos na sala virtual. Essas características associadas a apatia dos alunos, a falta de dinâmica, podem gerar um baixo desempenho, tendo como consequência, muitas vezes, as evasões. (COSTA et al., 2021).

Devido as dificuldades encontradas, surge nas universidades públicas e privadas a necessidade de desenvolvimento de maneiras alternativas de ensino, como as tentativas de adaptação e implementação de sistemas digitais. Essas tentativas, por sua vez, acabam por expor várias problemas, como: o acesso limitado ou inexistente dos estudantes e docentes às tecnologias necessárias, a sobrecarga de trabalho atribuída aos professores, a possível diminuição na qualidade no ensino, resultante da falta de planejamento de atividades em meios digitais devido a limitação de conhecimento tecnológico dos professores, o descontentamento dos estudantes e a falta de suporte psicológico aos docents. (GUSSO et al., 2020).

É notória a expansão das atividades docentes, em especial nas universidades públicas. Além de planejar, ministrar aulas e corrigir provas, habitualmente suas atividades envolvem: participar de reuniões, elaborar e preencher relatórios e formulários, organizar e coordenar atividades, assumir funções de gestão, elaborar e gerenciar 
projetos de pesquisa e de extensão, publicar trabalhos, responder e-mails institucionais, orientar alunos, atender solicitações da instituição e alunos, participar de comissões, prestar consultorias, participar de eventos da área de atuação, dentre outras variadas atividades. As diversas atribuições impostas, muitas vezes ultrapassam a sua carga horária. (CAMPUS; VÉRAS; ARAÚJO, 2020).

Ressalte-se que embora as atividades de ensino regulares não estivessem ocorrendo nas instituições devido a pandemia, as demais atividades de pesquisa, extensão e administrativas continuaram, presencialmente ou de modo remoto. (GUSSO et al., 2020). Além disso, a necessidade de dar seguimento ao ano letivo e considerando o tripé universitário do ensino, pesquisa e extensão, os docentes universitários precisam se adaptar, juntamente com suas rotinas de casa, considerando que precisam dar conta também das atividades domésticas. (KAPPES et al., 2021).

Vale destacar que com a diminuição de frequência de alunos e como as aulas passaram a ser remotas, turmas maiores foram disponibilizadas para uma quantidade menor de docentes. Considerando esse fator aliado a crise do país, muitas demissões aconteceram e/ou redução na contratação de novos profissionais para as universidades, devido a necessidade do corte de gastos. (COSTA et al., 2021).

Assim, deve-se reconhecer que uma nova proposta educativa não surge através de improviso. Mesmo considerando que é uma situação atípica, a mudança para o ERE deveria abranger também responsabilidades e reorganizações institucionais e governamentais por meio de ações que envolvam planejamento e capacitação dos profisisonais envolvidos. Entretanto, a pandemia da COVID-19 evidenciou que a virtualização do ensino tem sido protagonizada pelos professores, independentemente de suas dificuldades, incertezas, medo, ansiedade, depressão e sobrecarga de trabalho. Mesmo com as dificuldades, esses profissionais tem transformado e ressignificado a sua práticade forma criativa, com muito comprometimento e responsabilidade. (SANTOS; SILVA; BELMONTE; 2021).

\section{SAÚDE MENTAL DOS DOCENTES UNIVERSITÁRIOS}

A Organização Internacional do Trabalho (OIT) indica que desde 1983 a classe docente é a segunda maior categoria profissional, em nível mundial, a ser acometida por doenças de caráter ocupacional, incluindo reações alérgicas, distúrbios vocais, gastrite e 
até esquizofrenia (PEREIRA; SANTOS; MANENTI, 2020). Dessa forma, vale ressaltar a importância do cuidado com a saúde, em especial a saúde mental dos educadores, não apenas em detrimento da vulnerabilidade que se fez diante do cenário da COVID-19 e às condições de trabalho em formato home office, mas também devido a todas essas questões existentes anteriormente à pandemia, que causam preocupação devido ao impacto da falta de planos de ação e estratégias ao retorno das aulas presenciais. (KAPPES et al., 2021).

Nesse sentido, a pandemia deixou evidente que a educação brasileira apresentava algumas lacunas, por isso esse segmento acabou se tornando uma das mais afetadas, sendo assim é necessário avaliar os desafios que o corpo docente está enfrentando desde o início das aulas remotas (COSTA et al., 2021). Por consequência desta mudança tão importante no âmbito educacional, a OMS já reforçou que houve um aumento dos relatos de ansiedade e estresse entre profissionais de diversas áreas de ensino. (SILVA; BATISTA; TROTTA, 2020).

As alterações realizadas impactaram diretamente a forma de trabalho dos docentes em todos os níveis de ensino. Além disso, somam-se as preocupações de ordem familiar e pessoal, preocupações com a própria saúde e para com familiares resultando em evidentes impactos individuais e coletivos. (MONTEIRO; SOUZA, 2020). Por esse motivo e devido a este novo cenário de isolamento social, surgiram várias preocupações, não somente quanto ao prejuízo em relação à aprendizagem dos conteúdos curriculares, mas também quanto aos impactos que o isolamento poderia causar na saúde dos envolvidos. Para compreendermos as consequências do isolamento social na vida dos nossos alunos e professores, é relevante lembrar que a escola e as Universidades não podem ser reconhecidas como um espaço utilizado apenas para transmissão de conhecimento, mas também de convivência e de sistematização social. (SILVA; BATISTA; TROTTA, 2020).

Nesse sentido, a missão do professor foi expandida ao longo dos anos para além das dimensões da sala de aula para garantir a conexão entre comunidades e escolas. (COSTA et al., 2021). Neste contexto, o tempo que o professor necessita dispor para cumprir suas atividades também tem sido alterado e, ao mesmo tempo em que várias atividades são facilitadas com a introdução de novas tecnologias, existe a necessidade de maior dedicação, havendo um prolongamento no tempo de permanência do docente no exercício de sua profissão tanto dentro do ambiente de trabalho, como na dedicação ao planejamento e outras atividades. Diante do exposto, nota-se que a docência possui 
características peculiares em relação à organização do trabalho, e já é consenso na literatura que ela é causadora de estresse desse ambiente. (SOARES; MAFRA; FARIA, 2019).

Assim, deve-se compreender que o trabalho é vital para a vida humana, mas que, ofertado em situações precárias produz adoecimento. Além disso, considerando as circunstâncias relacionadas a redução de custos que está acontecendo nas Universidades públicas e privadas, evidencia-se que a precarização atinge principalmente o trabalho dos docentes das universidades públicas. Desse modo, para analisar as doenças e formas de adoecimento desses profissionais, há que se ponderar que a ideia de estabilidade no trabalho, cedeu lugar à competição, à luta cotidiana por reconhecimento, à sobrecarga de atividades e à obrigação por cumprir e conformar-se às regras, o que os coloca em uma posição de vulnerabilidade. (OLIVEIRA; PEREIRA; LIMA, 2018).

Outro aspecto relevante que não deve ser desprezado é o quão o ambiente também afeta a saúde mental desses professores, que antes do isolamento ocorria uma interação aluno/professor, em momentos informais, como trocas de ideias, desenvolvendo um ambiente leve de ensino. Mas com a pandemia essa interação se perdeu, com as câmeras desligadas, e na maior parte do tempo, os áudios também, o meio virtual passou a ter, em sua maioria, somente o professor falando. (COSTA et. al., 2021).

Com essa realidade, podemos dizer que o que iria talvez ocorrer na educação em uma década acabou acontecendo de forma "emergencial" em poucos meses. (BEHAR, 2020). Sendo assim, é possível notar um indicador ascendente no processo de adoecimento entre os docentes nas últimas décadas, especialmente no quesito de sofrimento relacionados às condições de trabalho. O sofrimento mental se dá por meio de um conjunto de manifestações do corpo e da psique, como estresse, ansiedade, depressão e fadiga. Fica evidente que esta realidade se intensificou com a pandemia e a precariedade ao cuidado da saúde física e mental deste público. (KAPPES et al., 2021).

\section{RESULTADOS E DISCUSSÃO}

Diante do que foi observado, a forma intempestiva como se deu a implementação do Ensino Remoto Emergencial fez com que as Instituições de Ensino reformulassem os seus processos de ensino, buscando novas estratégias para dar continuidade ao calendário letivo. Contudo, para a execução das atividades no novo formato entende-se que seria 
necessário ser disponibilizada formação para os docentes, bem como uma melhor infraestrutura, suporte técnico e pedagógico para que o trabalho dos professores pudesse ser desenvolvimento da melhor forma possível. (COSTA et al., 2021; KAPPES et al., 2021; SILVA, et.al., 2020; SILVA; BATISTA; TROTTA, 2020; OLIVEIRA; PEREIRA; LIMA, 2018; MONTEIRO; SOUZA, 2020; PEREIRA; SANTOS; MANENTI, 2020).

Dessa forma, já é possível perceber nas discussões que muitas são as reflexões atualmente sobre o quanto a escola exerce um papel fundamental na vida dos alunos, sobre a importância do papel do professor como mediador e a da necessidade dos alunos atuarem como protagonistas no processo de aprendizagem, além da possibilidade de avaliar a relevância da formação inicial e continuada com relação às novas tecnologias utilizadas no ensino. (AVELINO; MENDES, 2020; BEHAR,2020; PEREIRA; SANTOS; MANENTI, 2020).

Evidencia-se assim, que a adaptação ao novo modelo de ensino fez emergir a necessidade de discussões referentes a valorização docente, a de qual seria o verdadeiro papel da escola em pautas importantes como saúde, ações sociais, apoio aos alunos e profissionais da área de educação, a importância do fortalecimento das relações socioprofissionais no processo de ensino-aprendizagem, bem como a necessidade de planejamento por parte dos gestores referente ao plano de ação para amenizar os impactos econômicos, sociais e emocionais, que estão surgindo ou surgirão na retomada das aulas presencias, provocados pela pandemia da COVID-19. (AVELINO; MENDES, 2020; SILVA; BATISTA; TROTTA, 2020).

Considerando estes aspectos, destaca-se a necessidade de adaptações nos sistemas de ensino neste momento de pandemia. Percebe-se que a possibilidade de aguardar o fim da panemia para pensar na reposição do calendário acadêmico não parece mais ser uma opção. Isso porque pesquisas mais recentes, sugerem o prolongamento de quarentenas ainda para os próximos dois anos. (GUSSO et al., 2020).

Nesse sentido, os assuntos que estão sendo discutidos entre os estudiosos transitaram pelos desafios e impactos do formato de ensino remoto abrupto, cansaço, estresse e ansiedade aumentados e jornada estendida de trabalho. Estes avaliam o impacto que o contexto do trabalho remoto pode causar na saúde desse profissionais. Relatam também a relação de sofrimento e instabilidade emocionou dos docentes em decorrência da forma como esse trabalho vem sendo executado. (KAPPES et al., 2021; SILVA, et. 
al.; 2020; SANTOS; SILVA; BELMONTE, 2021; PEREIRA; SANTOS; MANENTI, 2020).

Além disso, enfatiza-se a importância de considerar nas discussões que existem realidades distintas entre docentes no Ensino Superior Público e nas Instituições particulares, bem como dos docentes que atuam apenas na graduação e os que atuam também na pós-graduação, sendo assim, deverão ser avaliadas as caracteríticas de cada contexto nas instituições visando proporcionar meios para um melhor desempenho por parte desses profissionais para a realização de suas atividades. (SILVA; ESTRELA; LIMA; ABREU; 2020).

A sobrecarga dos docentes universitários, visto que acumulam diversas atividades, e estas aumentaram ainda mais considerando essa nova realidade, visto que o tempo dedicado ao trabalho já não era suficiente, fazendo com que eles realizassem suas atividades de trabalho em casa, não conseguindo gerenciar muitas vezes, o tempo que deveria ser dedicado ao lazer, às atividades físicas, as atividades domésticas e ao convívio familiar, o que aumenta seus níveis de ansiedade e o risco de doenças. (SOARES; MAFRA; FARIA, 2019; SANTOS; SILVA; BELMONTE, 2021; BARROS DOS SANTOS JUNIOR; MONTEIRO, 2020; SILVA; BATISTA; TROTTA, 2020).

Nesse sentido, a responsabilidade da gestão diante das dificuldades apresentadas aumenta, pois existe a necessidade de acompanhamento os docentes nas atividades profissionais, para auxiliar no planejamento do trabalho em home office, pensando na organização para realização das atividades e visando uma melhor adaptação das práticas de ensino, que a mesma esteja próxima e sensível para reconhecer as potencialidades e limitações desse processo, a fim de identificar antecipadamente indicativo de sofrimento psicoemocional e assim intervir para evitar agravamento do estado de saúde desses profissionais. (GUSSO, et al., 2020; SANTOS; SILVA; BELMONTE, 2021; PEREIRA; SANTOS; MANENTI, 2020).

Considerando que possa existir a necessidade dos docentes dividirem suas angústias e na tentativa de diminuir os diversos sentimentos que permeiam suas vidas no contexto da pandemia, alguns autores sugerem formas de enfrentamento, onde as IES poderiam avaliar a possibilidade de disponibilizar espaços virtuais de escuta, compostos de equipes multiprofissionais, como uma forma de intervenção, para o atendimento a esses profissionais por meio de atividades voltadas para a saúde mental, com o intuito de reduzir as consequências do isolamento. (KAPPES et al., 2021; SANTOS; SILVA; 
BELMONTE, 2021; OLIVEIRA; PEREIRA; LIMA， 2018; SILVA; BATISTA; TROTTA, 2020).

Somando os impactos da pandemia a questões já existentes, como por exemplo, a fragilidade das políticas públicas em educação e as demandas extenuantes de trabalho no ensino, parece inevitável que decorram impactos negativos sobre a saúde desses profissionais. Os primeiros estudos nesta direção, procurando entender as consequências do cenário pandêmico, apontam especialmente para um sofrimento psíquico. (MONTEIRO; SOUZA, 2020; SILVA; ESTRELA; LIMA; ABREU.; 2020; SILVA; BATISTA; TROTTA, 2020; SOARES; MAFRA; FARIA, 2019; BARROS DOS SANTOS JUNIOR; MONTEIRO, 2020).

Considerando essas questões, ressalta-se a importância de orientar os docentes sobre a necessidade de reconhecer os seus próprios limites, aprender a lidar com novos desafios e organizar a rotina de modo a fazer um gerenciamento do tempo, como algo que pode ser positivo, pois proporciona que os indivíduos avaliem suas escolhas e hábitos de vida. Nesse sentido, o autocuidado advém do próprio indivíduo, mas também de rede de relações interpessoais que ele convive. (COSTA, et. al., 2021; BEHAR, 2020; FANTINI, et al., 2020).

De forma geral, ainda não se pode evidenciar os efeitos do Ensino Emergencial Remoto. É possível que consequências sejam percebidas nas instituições que adotaram esse formato ao longo dos semestres. Assim, é necessário pensar em alternativas para que seja evitado que as salas de aula das Universidades fiquem lotadas. Dadas as condições institucionais e as condições dos agentes envolvidos nesse processo, como gestores, incluindos os funcionários, professores e alunos nessa discussão, definirem quais serão as prioridades, no sentido de estabelecer o que precisa ser garantido no Ensino Superior durante o período de pandemia e o que seria viável e adequado ser ensinado nesse momento para evitar uma sobrecarga para os docentes e discentes. Esse talvez seja um dos pontos de partida para tornar o ensino emergencial mais planejado e estruturado visando a preservação da saúde dos profissionais envolvidos (GUSSO et al., 2020; PEREIRA; SANTOS; MANENTI, 2020).

\section{CONSIDERAÇÕES FINAIS}

O presente estudo se propôs compreender os impactos da implementação do Ensino Remoto Emergencial na saúde dos docentes universitários, a partir da investigação 
das possíveis limitações e dos desafios enfrentados pelos docentes para realização desse modelo de ensino diante do cenário atual de contaminação pelo coronavírus, visando despertar a discussão para possíveis estratégias de enfrentamento frente ao cenário devastador da COVID-19.

Entende-se que o Ensino Remoto Emergencial não é uma modalidade de ensino, não foi criado com bases e projeções pedagógicas com finalidades expressas e resultados delineados que sirvam de base para mensurar a qualidade educativa ao longo do tempo. Falta-lhe um arcabouço metodológico que possibilite uma didática que produza frutos, um sistema de avaliação e gestão coerente com o tempo de aprendizado.

Sendo assim, com base na análise dos artigos selecionados para este estudo, atingiu-se a proposta inicial, pois foi possível avaliar como está sendo impactante o isolamento social e a realização das atividades de forma remota para grande parte dos educadores, não apenas considerando as dificuldades pedagógicas e estruturais para realização das suas atividades na vida profissional, mas também para o estado socioemocional, considerando que cada sujeito possui características muito peculiares considerando esse aspecto. Portanto destaca-se que existe a necessidade de falar sobre estratégias para essa categoria, além de buscar conhecer quais são os recursos utilizados por esses profissionais para defender-se dos riscos existentes no ambiente de trabalho ou relacionados ao trabalho.

É importante que os gestores das Universidades, além de entederem os graves riscos biológicos provocados pela pandemia da COVID-19, percebam que existem outros riscos que interferem no bem-estar físico e mental dos docentes, pois, atualmente não se sabe ao certo como será o retorno das atividades e quais serão as consequências para a educação após o fim do isolamento social. Assim, a partir das discussões estabelecidas percebe-se a necessidade de avaliar a atual situação dos docentes diante das condições de trabalho para atuação no ERE e despertar a reflexão para que os gestores das Instituições de Ensino reavaliem como está sendo fornecido o suporte pedagógico, estrutural e tecnológico para estes profissionais.

\section{REFERÊNCIAS}

AVELINO, W. F.; MENDES, J. G. A realidade da educação brasileira a partir da

COVID-19. Boletim Conjuntura (BOCA) (UFRR), v. 2, p. 56-62, 2020. 
BARROS DOS SANTOS JÚNIOR, V.; MONTEIRO, J. C. S. Educação e COVID-19: as tecnologias digitais mediando a aprendizagem em tempos de pandemia. Revista Encantar, v. 2, p. 01-15, 2020.

BEHAR, P. A. O Ensino Remoto Emergencial e a Educação a Distância. Jornal da Universidade UFRGS, Porto Alegre, p. 1 - 1, 02 jul. 2020.

BRASIL. Portaria N$^{\circ}$ 343, de 17 de março de 2020. Dispõe sobre a substituição das aulas presenciais por aulas em meios digitais enquanto durar a situação de pandemia do Novo Coronavírus - COVID-19. D.O.U 18/03/2020. Disponível em: $<$ http://www.in.gov.br/en/web/dou/-/portaria-n-343-de-17-de-marco-de-2020248564376>. Acesso em: 03 out. 2021.

BRASIL(a). Lei no 9.394, de 20 de dezembro de 1996. Estabelece as diretrizes e bases da educação nacional. Disponível em: <http://www.planalto.gov.br/ccivil_03/Leis/L9394.htm>Acesso em:12 dez. 2021.

CAMPOS, T. C.; VÉRAS, R. M.; ARAÚJO, T. M. de. Trabalho docente em universidades públicas brasileiras e adoecimento mental: uma revisão bibliográfica.

Revista Docência do Ensino Superior, Belo Horizonte, v. 10, e015193, p. 1-19, 2020 DOI: https://doi.org/10.35699/2237-5864.2020.15193.

CARVALHO, E. M. dos S.; ARAUJO, G. C. Ensino remoto, saberes e formação docente: uma reflexão necessária. REVISTA COCAR (ONLINE), v. 14, p. 1-19, 2020 .

COSTA, B. R.; CARVALHO, K. G.; SILVA, E. L.; FERREIRA, M. F.; SOUZA, S.; HERNANDES, L. F.; PINHEIRO, M. D. S. L. B. Impacto da COVID-19 na saúde mental de educadores do ensino superior. In: I Congresso Inter. de Psicologia da Faculdade América, 2021, Cachoeiro de Itapemirim. Anais [...]. Manhaçu: Revista Pensar Acadêmico, 2021. v. 1. p. 1-1.

FANTINI, A. J. E.; MACEDO, D. C. F.; SOUZA, M. A. P.; OSCAR, S. G. F.; RIBEIRO, S. C. S. Universidade Federal de Minas Gerais (UFMG). Departamento de Atenção à Saúde do Trabalhador (DAST). Manual - Cuidando da saúde no trabalho remoto - Orientações Ergonômicas sobre Ergonomia. Minas Gerais: 2020. Disponível em:〈https://drive.google.com/file/d/1JPzy-IUxPB3yDIjYSSjfWGwrRwMic3YZ/view〉. Acesso em: 17 out. 2020

GOMES, N. P.; CARVALHO, M. R.S.; SILVA, A.F; MOITA, C. E.; SANTOS, J. R. L.; COUTO, T. M.; CARVALHO, L. C.; ALMEIDA, L. C. G. Saúde mental de docentes universitários em tempos de COVID-19. SAÚDE E SOCIEDADE (ONLINE), v. 30, p. 1, 2021.

GUSSO, H. L.; ARCHER A. B.; LUIZ, F. B.; SAHAO, F. T.; LUCA, G. G.; HENKLAIN, M. H. O.; PANOSSO, M. G.; KIENEN, N.; BELTRAMELLO, O.; GONCALVES, V. M. Ensino superior em tempos de pandemia: diretrizes à gestão universitária. EDUCAÇÃO \& SOCIEDADE, v. 41, p. 1-26, 2020. 
KAPPES, S.; SCHABAT, F. M.; ZOLETTI, G. K.; COMUNELLO, M. J.; BUSATO, M. A.; ROMAN JUNIOR, W. A. Saúde mental de docentes no cenário da pandemia da COVID-19. Congresso Internacional em Saúde. v. 8, 2021. Disponível em:< https://publicacoeseventos.unijui.edu.br/index.php/conintsau/article/view/19081/17814> Acesso em: dez. 2021)

MINISTÉRIO DA SAÚDE. Painel Coronavírus (COVID - 19). 2020. Disponível em: <https://covid.saude.gov.br/>. Acesso em: 03 out. 2020.

MONTEIRO, B. M. M.; SOUZA, J. C. Saúde mental e condições de trabalho docente universitário na pandemia da COVID-19. Research, Society andDevelopment. v. 9, n.9, p. 1-16, 2020.

PEREIRA, H. P.; SANTOS, F. V.; MANENTI, M. A. Saúde mental de docentes em tempos de pandemia: os impactos das atividades remotas. Boletim de conjuntura (BOCA). v. 3, n. 9, p. 1-9, 2020.

OLIVEIRA, A. S. D.; PEREIRA, M. S.; LIMA, L. M. Trabalho, produtivismo e adoecimento dos docentes nas universidades públicas brasileiras. Revista Brasileira de Psicologia Escolar e Educacional, 2018.

SANTOS, G. M. R. F.; SILVA, M. E.; BELMONTE, B. R. COVID-19: ensino remoto emergencial e saúde mental de docentes universitários. Rev. Bras. Saúde Matern. Infant., Recife, 21 (Supl. 1): S245-S251, fev., 2021

SILVA, A. F.; ESTRELA, F. M.; LIMA, N. S.; ABREU, C. T. A. Saúde mental de docentes universitários em tempos de pandemia. PHYSIS. Revista de saúde coletiva (online), v. 30, p. 300216-300216, 2020.

SILVA, P. F. T.; BATISTA, A. A. R.; TROTTA, L. M. Impactos na saúde socioemocional dos educadores durante a pandemia de COVID-19. Revista Carioca de Ciência, Tecnologia e Educação (online). v.5, n. especial, p. 80-82, 2020.

SOARES, M. B.; MAFRA, S. T.; FARIA, E. R. Fatores associados à percepção de estresse em docentes universitários em uma instituição pública federal. Revista brasileira de medicina do trabalho, v. 17, p. 90-98, 2019.

Recebido em: 08/03/2022

Aprovado em: 05/03/2022

Publicado em: 03/02/2022 